\title{
Neuropsychiatric complications after living donor liver transplantation: a prospective case series in an Egyptian center Mohamed A. Sakr ${ }^{a}$, Mohamed K. El-Naggar ${ }^{\mathrm{a}}$, Mohamed O. Khalifa ${ }^{\mathrm{a}}$, Hend M. Hussein ${ }^{a}$, Azza A. Abd El-Aziz ${ }^{b}$, Tarek A. Ahmed ${ }^{b}$, Mahmoud S. El-Meteini ${ }^{\mathrm{C}}$
}

Departments of ${ }^{\mathrm{a}}$ Tropical Medicine,

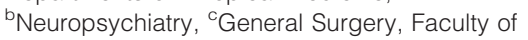
Medicine, Ain Shams University, Cairo, Egypt

Correspondence to Mohamed Omar Khalifa, MD, Department of Tropical Medicine, Ain Shams University, Cairo, 11566, Egypt. Tel: +20 222871 187; fax: +20 226820 665; e-mail: dmohammedomar76@med.asu.edu.eg

Received 16 June 2018

Accepted 8 July 2018

The Egyptian Journal of Internal Medicine 2018, 30:239-248

\begin{abstract}
Introduction and aim
Neuropsychiatric complications that develop after living donor liver transplantation (LT) are frequently encountered, though not adequately estimated among Egyptian recipients. We aimed to estimate the frequency of neuropsychiatric manifestations and neurocognitive functional changes after living donor LT.
\end{abstract}

Patients and methods

A prospective observational cohort study was conducted to evaluate 30 adult patients with end-stage liver disease preoperatively in a single Egyptian Transplant Center from November 2012 till January 2014. Relevant preoperative, intraoperative, and postoperative data were recorded and recollected and at 3 and 6 months of follow-up. The assessment included neurologic evaluation, Child-Turcotte-Pugh score, model of end-stage liver disease score, clinical hepatic encephalopathy staging scale, West Haven criteria, and International Society for Hepatic Encephalopathy and Nitrogen Metabolism score for semiquantitative assessment of encephalopathy.

\section{Results}

Hepatitis C virus was the main etiology for liver disease in 27 (90\%) patients. Overt hepatic encephalopathy was seen in $26.6 \%$, whereas covert hepatic encephalopathy occurred in $43.3 \%$. Postoperatively, $76.7 \%$ of the patients demonstrated neuropsychiatric manifestations, with $50 \%$ of them showing mainly early major events, namely, encephalopathy. Late minor manifestation rates were $50 \%$. Early cyclosporine administration and cold ischemia time longer than $40 \mathrm{~min}$ were significant predictors of occurrence of early neurologic events postoperatively ( $P=0.031$ and 0.025 , respectively). Both risk factors were associated with earlier and higher rates of neurologic complications.

\section{Conclusion}

Patients of living donor LT are at increased risk of developing early postoperative major neurologic sequelae, which become of less clinical significance later on. The patients' cognitive functions improve with time after transplant.

\section{Keywords:}

calcineurin inhibitors, cognitive function, encephalopathy, neurologic sequelae

Egypt J Intern Med 30:239-248

(1) 2019 The Egyptian Journal of Internal Medicine

1110-7782

\section{Introduction}

Liver transplantation (LT) is the only curative treatment in patients with end-stage liver disease [1]. Since the introduction of immunosuppressive agents for clinical use, the survival rates have increased from $20 \%$ to nearly $80 \%$ [2].

Although a functioning allograft will generally improve neurological impairment in those patients with certain pre-existing metabolic encephalopathy, clinical series have documented neurological complications in $8.3-47 \%$ of all patients receiving LT [3].

Earlier reports indicated that patients with neurologic sequelae (NS), especially those occurring early after surgery, showed high mortality rates [4]. In addition, patients with NS have longer hospitalization [5], higher need of re-transplantation, more infections, and lower self-sufficiency and social reintegration than patients without NS [3]. Quite often, multiple co-existing risk factors are identified in such patients [6].

The introduction and the advent in immunosuppressive treatment, namely, the calcineurin inhibitors, in the post-transplant setting have led to the amelioration of acute rejection episodes. Oftentimes, a combination of

This is an open access journal, and articles are distributed under the terms of the Creative Commons Attribution-NonCommercial-ShareAlike 4.0 License, which allows others to remix, tweak, and build upon the work non-commercially, as long as appropriate credit is given and the new creations are licensed under the identical terms. 
Figure 1

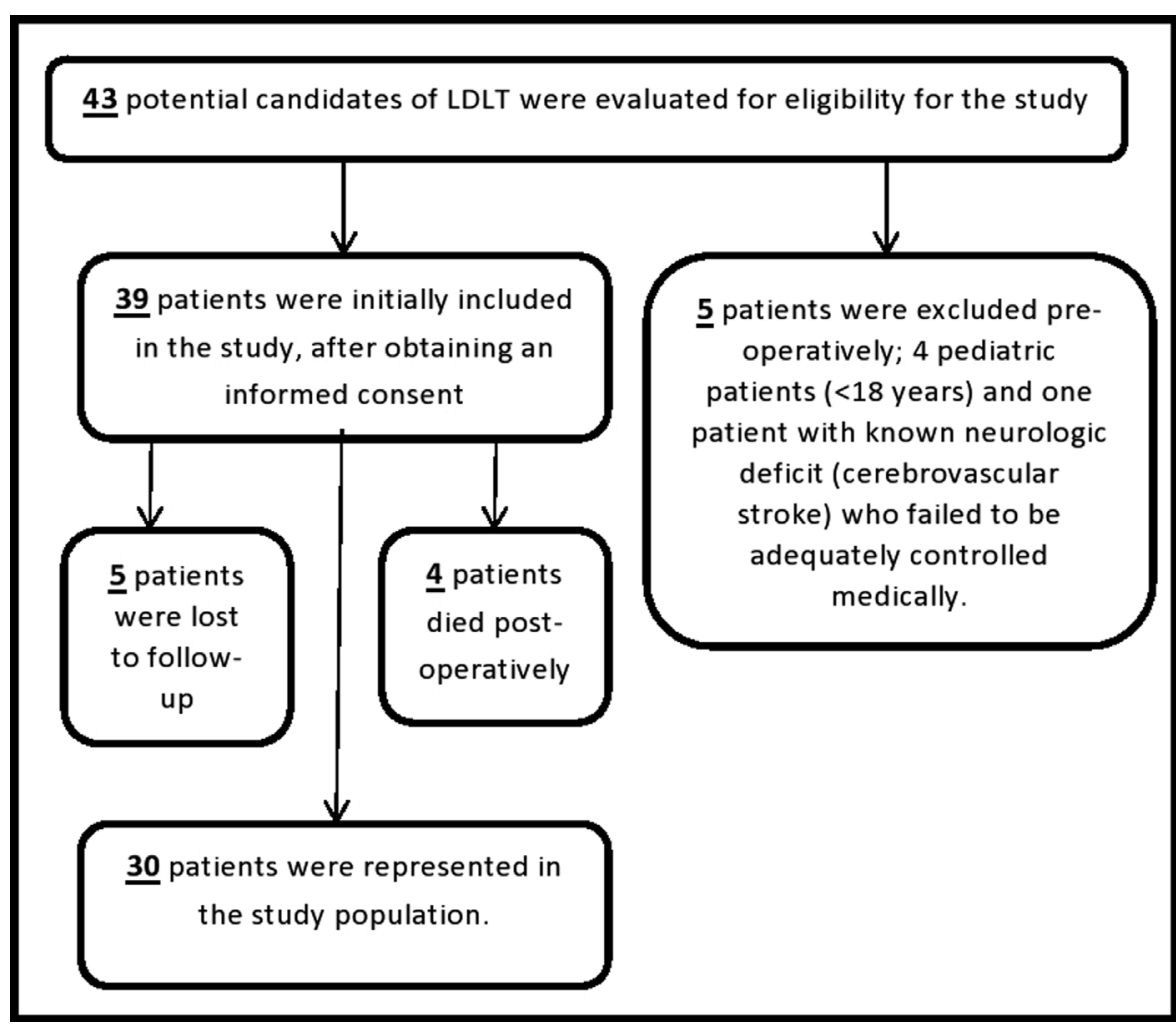

Flowchart of the evaluated patients for this study.

two or three different maintenance immunosuppressive drugs is used [7].

Paradoxically, NS are common following LT and are particularly relevant, as they affect up to a third of transplanted patients [8]. The majority $(\geq 75 \%)$ of these complications are observed within the first month after LT, suggesting a possible relationship between preoperative status and LT rather than the effect of immunosuppression [1].

We aimed in the present study to detect neuropsychiatric manifestations after living donor liver transplantation (LDLT) and investigate the possible role of certain preoperative, intraoperative, and postoperative risk factors.

\section{Patients and methods}

The study protocol was approved by the ethics committee of Faculty of Medicine, Ain Shams University (FMASU). Potential recipients of LDLT, presented to the Ain Shams Center for Organ Transplantation in Cairo from January 2012 through January 2014, were assessed for candidacy for LDLT according to the American Association for the Study of Liver Diseases (AASLD) practice guidelines [9].
The flowchart of the evaluated patients for this study is shown in Fig. 1.

The enrolled sample was subject to preoperative assessment [medical history, physical examination, laboratory and radiologic assessment for the etiology of hepatic disease, Child-Turcotte-Pugh score, and model of end-stage liver disease score (MELD) score], baseline neurologic assessment for primary co-morbid neurologic conditions (e.g. diabetic neuropathy), carotid Doppler of the neck veins, in addition to hepatic encephalopathy (HE) assessment and grading using West Haven criteria for semiquantitative grading of mental status [10] and Glasgow coma scale [11]. Whenever indicated by the neurologist, preoperative brain MRI and/or computed tomography and electroencephalogram were performed.

Applying standard surgical techniques and anesthesia protocols, the potential intraoperative risk factors were recorded. Post-operatively, all patients were managed in the same ICU.

All LT recipients were monitored for 6 months for postsurgical NS, which were either early (within 1 month after surgery) or late NS [12]. The neurologic and psychiatric assessment was carried 
out preoperatively and postoperatively by the same doctor for each patient. The neurologic event was considered 'major' if expected to have deleterious effect on survival, disability, expected ominous consequences, or expected to prolong hospital stay with greater total cost $[3,12]$.

Major events included persistent mental status alterations (e.g. owing to encephalopathy and cerebrovascular events), focal neurological signs (e.g. motor and/or sensory hemiparesis/hemiplegia, dysarthria/anarthria, and aphasia), myelinosis, cortical blindness, or seizures [13]. Minor events were those that disappeared spontaneously or were managed symptomatically, and had good prognosis. They included tremor, sleep disturbances, mood alterations, headaches, and paresthesia [12].

\section{Data management and statistical analysis}

Data were analyzed using IBM SPSS statistics version 22 (IBM Corp., Armonk, New York, USA) and MedCalc version 14 (MedCalc Software bvba, Ostend, Belgium). Numerical variables were presented as median and interquartile range, and intergroup differences were compared using the Mann-Whitney test. Categorical variables were presented as ratio or as number and percentage, and between-group differences were compared using Fisher's exact test.

Receiver operating characteristic curve analysis was used to examine the value of numerical variables for prediction of the occurrence of NS. Multivariable binary logistic regression analysis was used to determine independent predictors of NS. Explanatory variables that were significantly associated with the outcome of interest at a permissive significance level of $P$ less than 0.1 by univariate analysis were included in multivariable regression analysis, and the backward method was employed to build up the final model. For variables fulfilling the inclusion criterion of $P$ less than 0.1 but showing strong multi-colinearity, the more relevant variable was included, and the other less relevant variable was excluded from the model.

Time to event analysis was done using the Kaplan-Meier method. The significance of the results was assessed in the form of $P$ values as follows:

(1) Nonsignificant when $P$ value was greater than 0.05 .

(2) Significant when $P$ value was less than or equal to 0.05 .

(3) Highly significant when $P$ value was less than or equal to 0.01 .

\section{Results}

As per the underlying liver disease etiology, hepatitis $\mathrm{C}$ virus was responsible for $90 \%$ (27) of the cases. The remaining three $(10 \%)$ cases were found to have had documented positive $\mathrm{HBsAg}$ or DNA, autoimmune hepatitis, and cryptogenic cirrhosis. More than half of the patients $(56.7 \%)$ were of Child class $\mathrm{C}$, and the median MELD score was 15 (12.75-18). Only eight (26.6\%) of the recruits had grade II-III HE, whereas seven (23.3\%) participants had preoperative peripheral neuropathy. Carotid Doppler revealed insignificant hemodynamic changes in five $(15.6 \%)$ participants, whereas the electroencephalogram showed positive findings in four (13.3\%) patients only, in the form of temporoparietal epileptic activity with secondary generalization on top of average background in one patient and progressive slowing in rhythmic activity and triphasic waves in the rest. The rest of preoperative patient characteristics are demonstrated in Table 1.

The overall incidence of neurologic events after transplant amounted to $76.7 \%$ of the study participants at different points of follow-up. During the early ( 1 month after transplant) period, $50 \%$ of the

Table 1 Preoperative basal characteristics of the enrolled population $(n=30)$

\begin{tabular}{|c|c|}
\hline Variables & Value $[n(\%)]$ \\
\hline Age (years) & $52(48-56)$ \\
\hline Sex (male/female) & $29 / 1$ \\
\hline DM & $13(43.3)$ \\
\hline Hypertension & $3(10.0)$ \\
\hline Benign PVT & 7 (23.3) \\
\hline $\mathrm{HCC}$ & $15(50.0)$ \\
\hline Patients with data suggestive of HRS & $5(16.7)$ \\
\hline \multicolumn{2}{|l|}{ Child-Pugh score } \\
\hline Child A & $2(6.7)$ \\
\hline Child B & $11(36.7)$ \\
\hline Child C & $17(56.7)$ \\
\hline MELD score & $15(12.75-18)$ \\
\hline \multicolumn{2}{|l|}{ Encephalopathy grade (WHC) } \\
\hline Unimpaired & $9(30.0)$ \\
\hline Minimal HE & $8(26.7)$ \\
\hline Grade I HE & $5(16.7)$ \\
\hline Grade II HE & $7(23.3)$ \\
\hline Grade III HE & 1 (3.3) \\
\hline \multicolumn{2}{|l|}{ Preoperative neurological assessment } \\
\hline NAD & $22(73.3)$ \\
\hline PN & $7(23.3)$ \\
\hline CVI & $1(3.3)$ \\
\hline Abnormal MRI & $1(3.3)$ \\
\hline
\end{tabular}

$\mathrm{AlH}$, autoimmune hepatitis; CVI, cerebrovascular insufficiency; DM, diabetes mellitus; HCC, hepatocellular carcinoma; HRS, hepatorenal syndrome; MELD, model of end-stage liver disease; PN, peripheral neuropathy; PVT, portal vein thrombosis; WHC, West Haven criteria. 


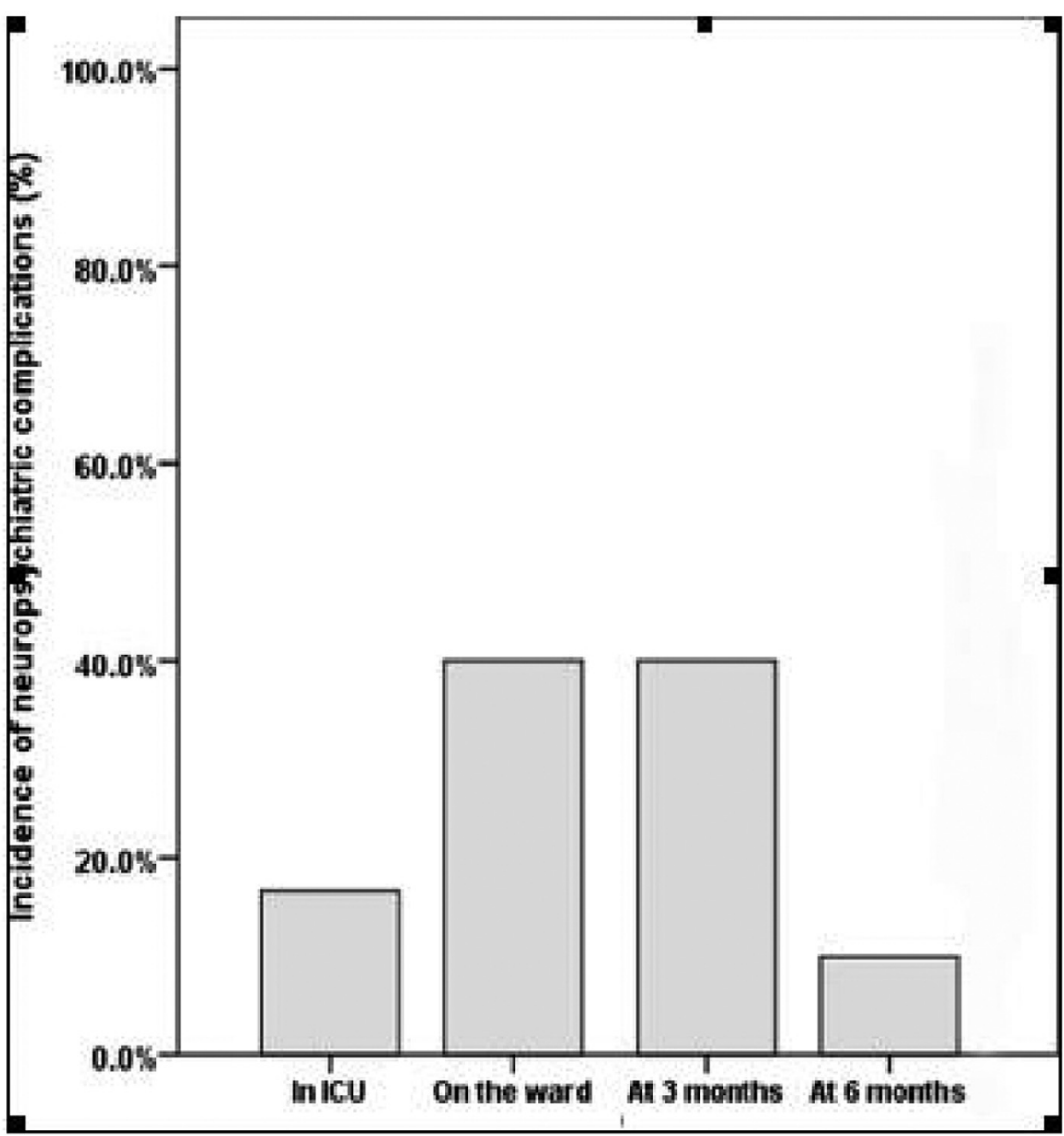

Incidence of neurologic sequelae in the whole study population throughout the postoperative period.

subjects (15 patients) were symptomatic for NS, where 43.3\% (13 patients) exhibited encephalopathy. The rest of the $6.6 \%$ (two patients) had minor events in the form of peripheral neuropathy and vertigo. At the time of development of early major NS, eight (26.7\%) patients were on CsA and four (13.3\%) were on $\mathrm{Fk}$, whereas one (3.3\%) patient was on neither, and his encephalopathy was attributed to cerebral edema. The median time to diagnosis of early NS was 17 days [9-17 at 95\% confidence interval (CI) for the median].

As per the rest of follow-up period, half of the patients experienced late exclusively minor events. Of which, the frequencies of peripheral neuropathy, depression, and tremors were $11(36.7 \%)$, three $(10 \%)$, and one (3.3\%), respectively. Of note, the incidence of such events was on a declining trend through time [i.e. 12 (40.0\%) at 3 months and three (10\%) at 6 months], as shown in Fig. 2.

None of the preoperative demographic characteristics, underlying liver disease or encephalopathy status or associated co-morbid conditions, was significantly different between those who demonstrated early NS and those who did not when univariate analysis was undertaken (Table 2).

Of the operative and postoperative factors as shown in Table 3, there was a statistically significant difference in the cold ischemia time (CIT), with longer median time of $45 \mathrm{~min}$ (40-90) among symptomatic cases rather than asymptomatic patients. Moreover, there were statistically significant lower proportions of patients who developed early NS while on Fk rather than asymptomatic subjects. Conversely, despite being statistically insignificant, the percentage of patients who developed early NS while on CsA was higher than those who were free.

Owing to the strong colinearity with early CsA administration, and apparently protective effect of Fk, Fk administration during early postoperative period was not counted as a predictor variable (correlation coefficient: $-0.934 ; P<0.0001$ ). Hence, simple binary logistic regression analysis illustrated that patients exposed to early CsA were at sixfold 
Table 2 Univariate analysis of preoperative, intraoperative, and postoperative patient characteristics $(\boldsymbol{n}=\mathbf{3 0})$

\begin{tabular}{|c|c|c|c|}
\hline Variables & No early NS $(n=15)[n(\%)]$ & Early NS $(n=15)[n(\%)]$ & $P$ value \\
\hline \multicolumn{4}{|c|}{ Preoperative patient characteristics } \\
\hline Age (years) & $52(48-57)$ & $50(47-56)$ & 0.724 \\
\hline Sex (male/female) & $14 / 1$ & $15 / 0$ & 1.000 \\
\hline DM & $8(53.3)$ & $5(33.3)$ & 0.461 \\
\hline Hypertension & $2(13.3)$ & $1(6.7)$ & 1.000 \\
\hline \multicolumn{4}{|l|}{ Etiology of cirrhosis } \\
\hline $\mathrm{HCV}$ & $15(100.0)$ & $12(80.0)$ & 0.224 \\
\hline HBV & $0(0.0)$ & $1(6.7)$ & \\
\hline AlH & $0(0.0)$ & $1(6.7)$ & \\
\hline Other cause(s) & $0(0.0)$ & $1(6.7)$ & \\
\hline $\mathrm{HCC}$ & $10(66.7)$ & $5(33.3)$ & 0.144 \\
\hline HRS & $2(13.3)$ & $3(20.0)$ & 1.000 \\
\hline Child score & $10(9-11)$ & $10(9-11)$ & 0.523 \\
\hline \multicolumn{4}{|l|}{ Child class } \\
\hline Child A & $1(6.7)$ & $1(6.7)$ & 0.772 \\
\hline Child B & $6(40.0)$ & 5 (33.3) & \\
\hline Child C & $8(53.3)$ & $9(60.0)$ & \\
\hline MELD score & $14(13-17)$ & $16(12-19)$ & 0.260 \\
\hline
\end{tabular}

$\mathrm{AlH}$, autoimmune hepatitis; DM, diabetes mellitus; HCC, hepatocellular carcinoma; HRS, hepatorenal syndrome; MELD, model of endstage liver disease; PVT, portal vein thrombosis.

Table 3 Comparison of patients with or without early neurologic sequelae regarding operative and postoperative characteristics $(n=30)$

\begin{tabular}{lccc}
\hline Variables & No early NS $(n=15)[n(\%)]$ & Early NS $(n=15)[n(\%)]$ & $P$ value \\
\hline Operative patient characteristics & & $10.0(9.0-12.0)$ & 0.149 \\
Operative time (h) & $9.5(8.0-10.0)$ & $1.00(0.90-1.20)$ & 0.883 \\
GRWR & $1.00(0.90-1.30)$ & $45(40-90)$ & 0.049 \\
CIT (min) & $40(30-45)$ & $45(30-60)$ & 0.801 \\
WIT (min) & $45(30-60)$ & $6(2-14)$ & 0.723 \\
Blood component usage (units) & $5(2-9)$ & $7(46.7)$ & 0.714 \\
Significant intraoperative PS shunting ${ }^{4}$ & $9(60.0)$ & $8(53.3)$ & 1.000 \\
Significant intraoperative hemodynamic instability & $7(46.7)$ & & \\
Postoperative patient characteristics & & $5(33.3)$ & 0.027 \\
Early Fk administration & $12(80.0)$ & $9(60.0)$ & 0.062 \\
Early CsA administration & $3(20.0)$ & $9(60.0)$ & 0.272 \\
Hypoxemia in early postoperative period & $5(33.3)$ & $10(66.7)$ & 1.000 \\
Documented systemic infections & $11(73.3)$ & $4(26.7)$ & 0.449 \\
Overall incidence of early surgical complications & $7(46.7)$ & $7(5-10)$ & 0.073 \\
ICU stay (days) & $5(5-6)$ & $24(20-30)$ & 0.545 \\
Hospital stay (days) & $24(19-26)$ & \\
\hline
\end{tabular}

CIT, cold ischemia time; PS shunting, portosystemic shunting; WIT, Warm ischemia time. ${ }^{\Delta}$ Significant intraoperative PS shunting; moderate to severe portosystemic shunts. 'Significant intraoperative hemodynamic instability; hypotension requiring norepinephrine, hypotension with bradycardia, ventricular fibrillation, hypertension requiring nitrates. Bold values are the significant values.

higher risk of developing early NS $(P=0.032,95 \% \mathrm{CI}$ : 1.17-30.73), which also tend to occur earlier than those who were not prescribed CsA (HR: 3.20; 95\% CI: $1.08-9.49, P=0.017)$.

Subsequent multivariable regression analysis was done for the statistically significant predictor variables as depicted in Table 4.

Applying CIT and early CsA administration could predict the occurrence of early NS with area under the curve of 0.71 (95\% CI: $0.52-0.86)$ and 0.7 (95\% CI: 0.506-0.853),
Table 4 Multivariable binary logistic regression analysis for predictors of early neurologic sequelae

\begin{tabular}{lccc}
\hline Variables & OR & $95 \% \mathrm{Cl}$ & $P$ value \\
\hline CIT (min) & 1.10 & $1.01-1.19$ & $\mathbf{0 . 0 2 5}$ \\
Early CsA administration & 38.14 & $1.40-1039.34$ & $\mathbf{0 . 0 3 1}$
\end{tabular}

$\mathrm{Cl}$, confidence interval; $\mathrm{CIT}$, cold ischemia time; OR, odds ratio. Bold values are the significant values.

respectively. CIT at a cutoff of $40 \mathrm{~min}$ had a higher sensitivity of $73.33 \%$ (95\% CI: 44.9-92.2, $P=0.031$ ), whereas early CsA administration demonstrated a higher specificity of $80 \% \quad(95 \%$ CI: 51.9-95.7, 


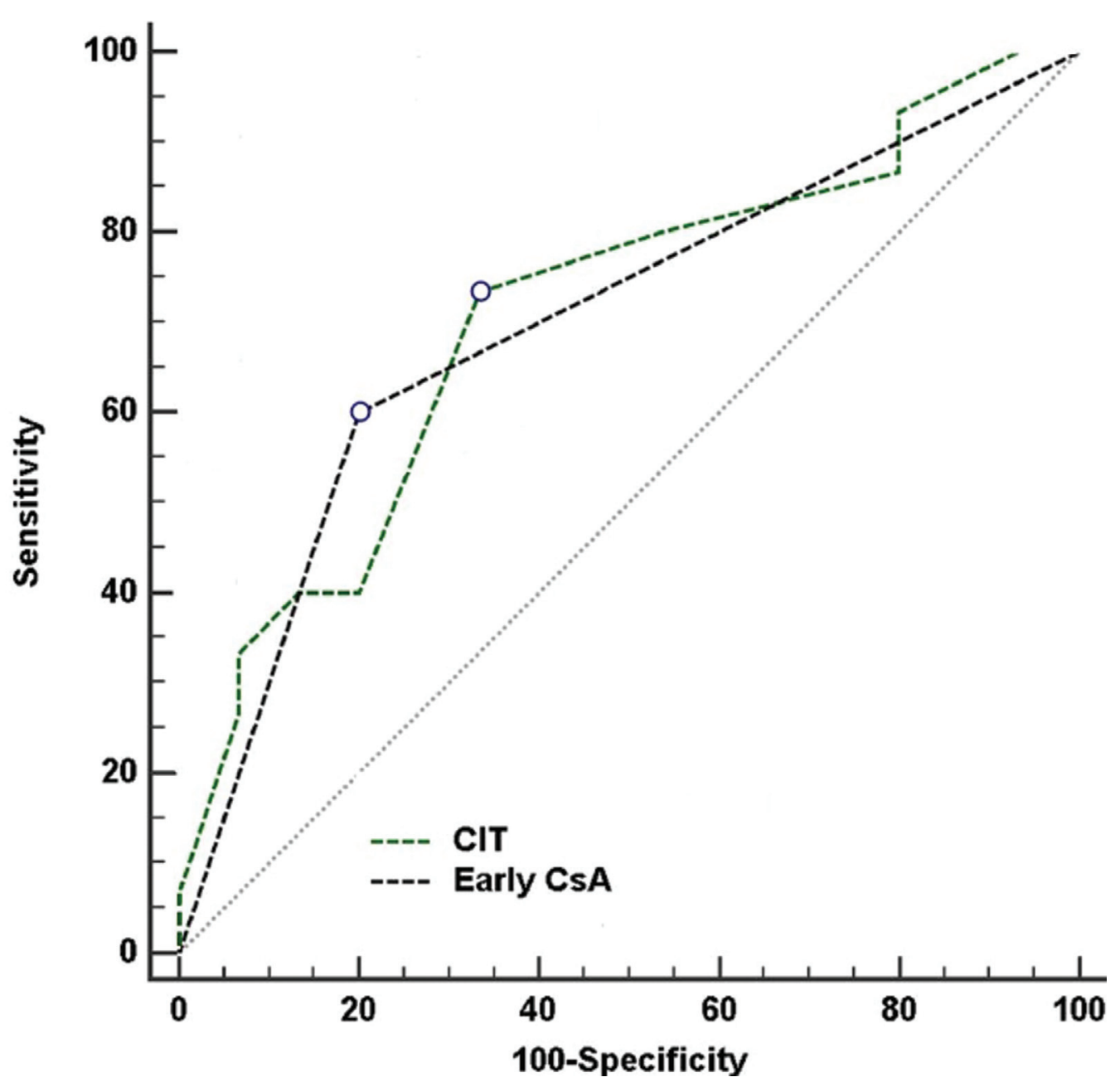

Comparison of the receiver operating characteristic curves for prediction of early neurologic sequelae using intraoperative cold ischemia time, and early CsA administration.

$P=0.018$ ), as shown in Fig. 3. Patients with CIT longer than 40 min or early recipients of $\mathrm{CsA}$ were found to be at significantly higher risk of developing early NS which tended to occur earlier than those who had shorterCIT and non-CsA-treated subjects (HR: 3.05; 95\% CI, 1.11-8.40, $P=0.041$ and HR: 3.20; 95\% CI: 1.08-9.49, $P=0.017$, respectively) (Figs 4 and 5, respectively).

\section{Discussion}

One of the countries most affected by hepatitis $\mathrm{C}$ virus is Egypt. The Egypt Demographic and Health Surveys (EDHS) measured antibody prevalence among the adult population aged $15-59$ years at $14.7 \%$ in 2009 [14] and at $10.0 \%$ in 2015 [15].

Our study population showed a relatively higher prevalence of pre-operative clinically overt $\mathrm{HE}$ (grades II-III) [30\%] than the previous study by Vizzini et al. [16] (14.3\%), but much less than Dhar et al. [5] (51.5\%). The high prevalence of preoperative
$\mathrm{HE}$ in our study may be attributed to the use of $\mathrm{HE}$ assessment and psychiatric evaluation of patients by a specialized psychiatrist; however, this was not performed in the other two studies.

While assessing the patients preoperatively for neurological disarray, it was found that the elicited peripheral neuropathy occurred in the clinical setting and hence explainable by the relatively high prevalence of diabetes mellitus (43.3\%) and hypertension (10\%) among the studied group.

Although most of the published reports that dealt with NS after LT were retrospectively led, the recent studies that included both LDLT and deceased donor liver transplantation (DDLT) and performed head-to-head comparisons were quite scarce and displayed varying data. Some concluded that there was a significantly higher difference in the incidence of NS in DDLT setting as Saner et al. [1], whereas some reported no effect, such as Kim et al. [17]. 


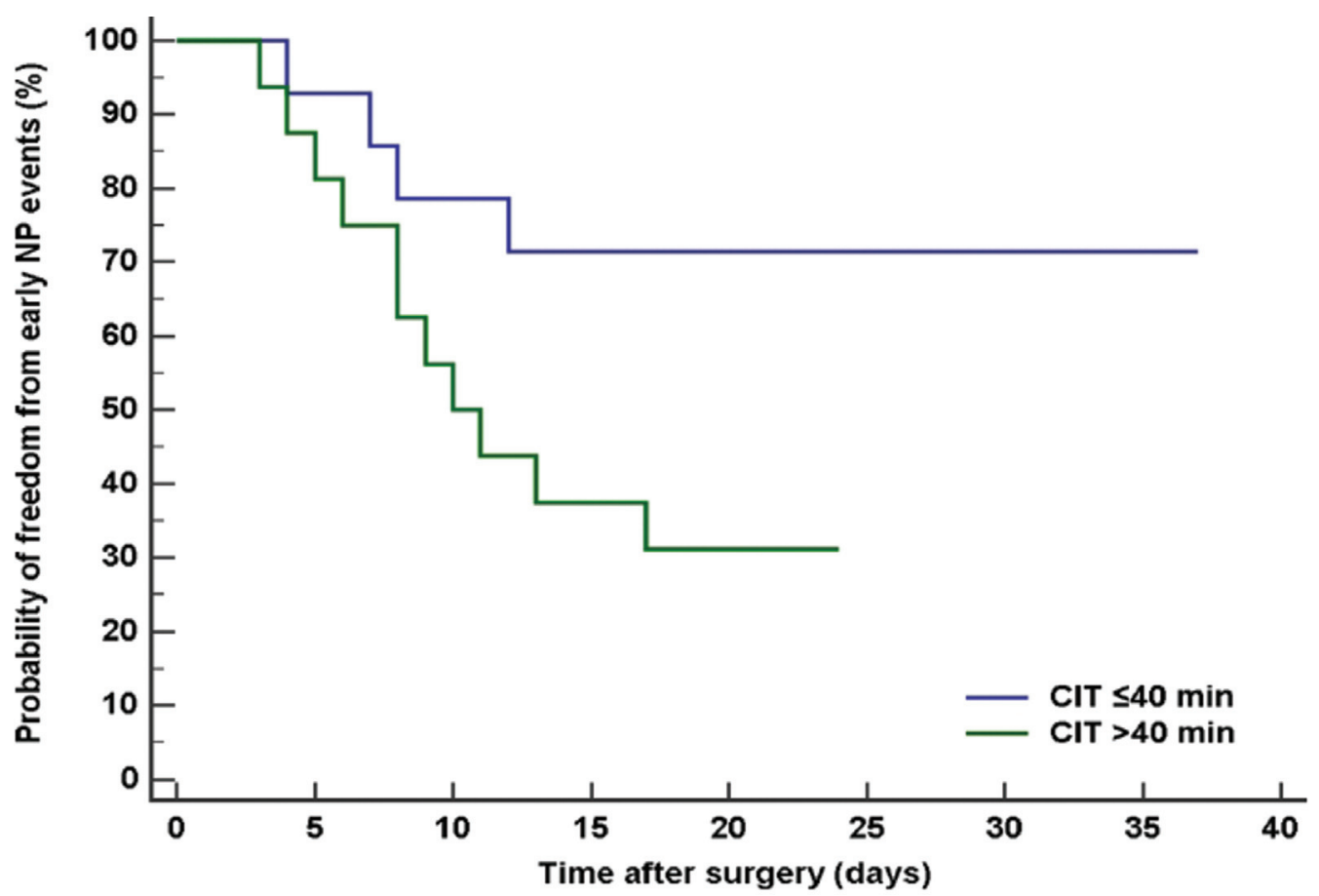

Kaplan-Meier curve for the time to development of early neurologic sequelae in patients with a cold ischemia time of less than or equal to 40 min or greater than $40 \mathrm{~min}$

Figure 5

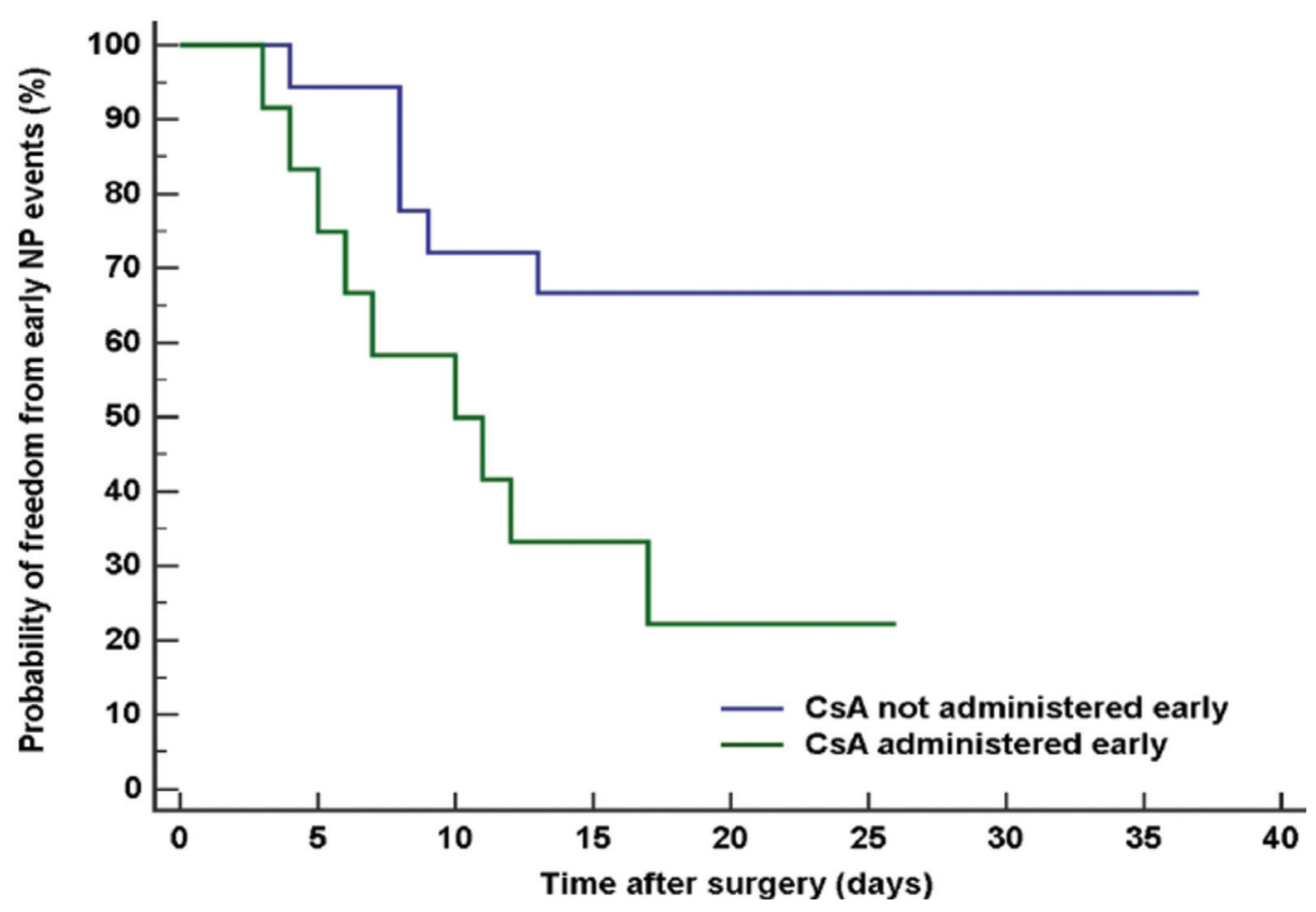

Kaplan-Meier curves for time to development of early neurologic sequelae in patients with or without early CsA administration.

The present study demonstrated a relatively high overall frequency of NS after LDLT (76.6\%). The early and late events, despite being of similar frequencies (50\%), those which occurred during the early postoperative period seemed to be of a substantially greater significance than those developed later because of the perceived greater clinical (namely, the hemodynamic and metabolic burden) together with the surgical burden on the transplant recipient on one side, and the medical 
transplant decision makers concerned with drug adjustment, withdrawal or shift in the face of adequate immune suppression on the other side. This was interpreted in the finding of $86.67 \%$ of the early NS of major effect, opposed to those later NS which were not only exclusively minor but on a downward trend too.

The current study results seem to go in accordance with the ranges stated by Ghaus et al. [18] (62\%) and Amin and Atta [19] (50.9\%) for the incidence of posttransplant NS. In the latter series, the first 110 Egyptian patients to undergo LDLT were reviewed during their ICU stay from May 2001 to April 2005. They concluded that the incidence of major NSs amounted to $32.7 \%$ of all LDLT patients [19], which, along with our study (43.3\%), depict a much greater frequency than that stated by Lewis and Howdle [3] (26\%) and Saner et al. [20] (24.7\%) and Vizzini et al. [16] (23.4\%).

In the current study, the major complications observed during the early period were all in the form of encephalopathy; which appeared in $43.3 \%$ of the transplant recipients. Delirium and acute confusion state were the chief presentations. The delirious state involved hallucinations, mostly visual and occasionally agitation episodes.

The cause of post-transplant encephalopathy in patients with a primary functioning graft remains unclear [1]. The underlying mechanisms are unknown, although in one large prospective study, the authors diagnosed a diffuse encephalopathy (anoxic, septic, or metabolic) as the most common complication occurring in $56.5 \%$ of $\mathrm{NS}$ in LT patients [21]. Its incidence varied widely among centers from 12 to $84 \%$ of patients $[1,3,5-7,20]$.

Postoperative encephalopathy was explained to be a manifestation of persistent major portosystemic shunts or immunosuppressant-associated toxicity by some [7].

Postmortem studies show that diffuse anoxic-ischemic changes were the main neuropathological findings, which could be the cause of encephalopathy [22].

In comparison with most of the aforementioned published studies, our series shows a complete absence of post-transplant CNS infectious complications, pontine myelinosis, myopathy, and cerebrovascular events. A considerable proportion of those who developed minor events, whether in the early or the late phase, had them in the form of peripheral neuropathy with sensory disturbance. A probable explanation of this relatively high frequency could be the considerable prevalence of diabetes among recipients before transplant that could have augmented their neuropathy risk.

Published reports on peripheral toxicity point out that it usually occurs weeks to months after starting immunosuppressive treatment. The more severe forms, as chronic inflammatory polyneuropathy, are the most commonly accounted for in medical literature and have been observed during Fk therapy [23]. In the study performed by Lewis and Howdle [3], only $0.7 \%$ of 402 patients complained of peripheral neuropathy. However, in a more recent series, it was deduced that the frequency of peripheral neuropathies after LT was as high as $28.6 \%$, in addition to motor disturbances, sensory disturbance and polyneuropathy at the rates of $18.4,8.2$, and $2 \%$, respectively [17]. None of the aforementioned studies, however, accounted for those patients' glycemic control or diabetes status.

It is worth noting that seizures were noticed in two patients after transplant, after the completion and termination of patient enrollment in the study. The scarcity of seizures incidence in our series is understandable, knowing that in the recent 10 years, the rates of seizures seem to have declined. The reported incidence was up to $20 \%$ [3], then $11.6 \%$ [20], 0\% [17], 8\% [5], 1\% [16] and 7.3\% [19] owing to the improvement in postoperative intensive care, accurate diagnosis and treatment of metabolic disorders, and the improved immunosuppressive drugs with fewer adverse effects [1].

Similar to our series, no correlation was observed between primary diagnosis and the incidence of NS as reported by Ghaus et al. [18], Saner et al. [20], Kim et al. [17], and Amin and Atta [19]. On the contrary, Lewis and Howdle [3] and Saner et al. [1] reported a higher rate of NS after LT for primary biliary cirrhosis, alcoholic cirrhosis, and autoimmune hepatitis.

We found that the occurrence of NS could not be explained by differences in the underlying functional state of the liver as indicated by MELD, Child scores, operative graft-recipient weight ratio or warm ischemia time between the groups, which goes in agreement with Saner et al. [1] Moreover, we found no difference between the early NS group and asymptomatic patients regarding preoperative overt $\mathrm{HE}$, which disagrees with Vizzini et al. [16] who deduced that $\mathrm{HE}$ in the immediate preoperative period was associated with high post-transplant neurologic complications. 
Among the operative factors in our sample, long CIT over 40 min could predict the occurrence of early postoperative NS. In fact, Saner et al. [20] deduced that the lower incidence of NS among the LDLT recipients was associated with the significantly shorter CIT than that of the DDLT. The reason for this phenomenon remains uncertain: it could be correlated with a better quality of the transplanted graft and the improved detoxification power in comparison with longer CIT [24]. This finding was not consistent when assessed on a homogenous group of LDLT recipients where the difference in CIT did not predict the occurrence of NS [1].

Regarding the clinical outcomes, those with early NS in our study had insignificant difference in ICU and overall hospital stay in comparison with the other group, which agrees with the more recent reports by Saner et al. [1] and defies older studies by Lewis and Howdle [3], Saner [20], Kim et al. [17], and Dhar et al. [5].

The discrepancy in results between studies, with respect to the estimated frequency and the description of the NS, could be attributed to the scarcity of the published studies exclusively performed on recipients to LDLT, so we had to include heterogeneous reports from studies on DDLT and LDLT subjects, tackled as single series, in our comparisons, such as Saner et al. [20], Kim et al. [17], and Vizzini et al. [16] No further elaboration of each group's distinct characteristics, disease description, or progress was reported. Other studies included those with pre-transplant fulminant hepatitis or re-transplantation recipients, despite their unique underlying conditions. In one study by Kim et al. [17], even patients who underwent simultaneous liver and kidney transplant were included in the analysis. Moreover, the retrospective study design in most of these studies could have added to this difference.

Missing data such as racial demographic characteristics (in some studies essentially performed on populations of varying ethnic backgrounds), the limited number of patients, the underrepresentation of patient categories, and the limited etiologic variability of the underlying liver disease were noticed in some studies, the current study included. All these factors contributed to further gapping of the different studies' results and rendered such results difficult to extrapolate to the actual transplant population. In fact studies with conditions similar to our study as those by Ghaus et al. [18] and Amin and Atta [19] tended to display less contradicting findings.

Dissimilar or unreported treatment protocol was a key feature of discrepancy as well. Vizzini et al. [16] adopted a steroid-free immunosuppression approach shortly before the end of the study, which was not taken into account with further risk stratification for the development of NS in their study, though, considered a substantial risk factor by some as Balderramo et al. [25] These observations had an unfavorable effect on the internal validity of such study results. Some studies, such as Lewis and Howdle [3], Dhar et al. [5], and Amin and Atta [19], did not sufficiently specify their immunosuppressive or the steroid treatment protocol.

On our side, the exclusion of those who failed to complete the follow-up period of 6 months might have undermined the brunt of other medical and surgical postoperative morbidities in the face of neuropsychiatric presentations.

Lastly, the lower incidence of NS in some studies can be attributed to the low-dose-based immunosuppressive treatment adopted by some centers. Kim et al. [17], for instance, initiated treatment at dose of $0.025 \mathrm{mg} /$ $\mathrm{kg} /$ day for $\mathrm{Fk}$, whereas the initial dose of cyclosporine was $1.5 \mathrm{mg} / \mathrm{kg} /$ day.

\section{Conclusion}

Neuropsychiatric sequelae after LDLT occur commonly among Egyptian transplant recipients, being present in $76.6 \%$ of the current series. The leading major neuropsychiatric complication reported was encephalopathy. Neuropsychiatric complications tended to decrease in frequency and clinical significance with time after transplant, indicating the short-lived and largely benign nature of these events.

Prolonged intra-operative CIT and postoperative CsA drug exposure are risk factors for the development of NS among adult LDLT recipients.

\section{Acknowledgements}

The authors thank the physicians who performed the transplant surgeries, the intensivists, and the rest of the transplant hepatologists involved in liver transplantation team at Ain Shams University Specialized Hospitals.

Concepts was done by Mohamed Amin Sakr, Mohamed Khairy El-Naggar and Mahmoud Shawky El-Meteini; Mohamed Amin Sakr, Mohamed Khairy El-Naggar, Mohamed Omar Khalifa, Hend Mubarak Hussein and Mahmoud Shawky El-Meteini done the design; definition of intellectual content was done by Azza Abd El-Nasser Abd El-Aziz and Tarek Asaad Ahmed; content was done by Mohamed Omar Khalifa, Hend Mubarak Hussein, Azza Abd El-Nasser Abd 
El-Aziz and Tarek Asaad Ahmed; literature search was done by Mohamed Omar Khalifa and Hend Mubarak Hussein; clinical studies was done by Mohamed Omar Khalifa, Azza Abd El-Nasser Abd El-Aziz and Tarek Asaad Ahmed; data acquisition was done by Hend Mubarak Hussein, Azza Abd El-Nasser Abd El-Aziz and Tarek Asaad Ahmed; data analysis was done by Mohamed Amin Sakr, Mohamed Khairy El-Naggar, Mohamed Omar Khalifa and Mahmoud Shawky ElMeteini; statistical analysis was done by Hend Mubarak Hussein, Azza Abd El-Nasser Abd ElAziz and Tarek Asaad Ahmed; manuscript preparation was done by Mohamed Amin Sakr, Mohamed Khairy El-Naggar, Mohamed Omar Khalifa, Hend Mubarak Hussein and Mahmoud Shawky El-Meteini; manuscript editing was done by Mohamed Amin Sakr, Mohamed Omar Khalifa, and Hend Mubarak Hussein; manuscript review was done by Mohamed Amin Sakr, Mohamed Khairy ElNaggar, Mohamed Omar Khalifa, Azza Abd ElNasser Abd El-Aziz, Tarek Asaad Ahmed and Mahmoud Shawky El-Meteini.

\section{Financial support and sponsorship \\ Nil.}

\section{Conflicts of interest}

There are no conflicts of interest.

\section{References}

1 Saner FH, Gensicke J, Olde Damink SWM, Pavlaković G, Treckmann J, Dammann $\mathrm{M}$, et al. Neurologic complications in adult living donor liver transplant patients: an underestimated factor? J Neurol J Neurol 2010; 257:253-258.

2 Starzl TE, Iwatsuki S, Esquivel CO, Todo S, Kam I, Lynch S, et al. Refinements in the surgical technique of liver transplantation. Semin Liver Dis 1985; 5:349-356.

3 Lewis MB, Howdle PD. Neurologic complications of liver transplantation in adults. Neurology 2003; 61:1174-1178.

4 Guarino M. Immunosuppressive therapy. Gastroenterol Int Congress Proc 1999; 12:140-145.

5 Dhar R, Young GB, Marotta P. Perioperative neurological complications after liver transplantation are best predicted by pre-transplant hepatic encephalopathy. Neurocrit Care 2008; 8:253-258.
6 Zivković SA. Neurologic complications after liver transplantation. World J Hepatol 2013; 5:409-416.

7 Teperman LW. Impact of pretransplant hepatic encephalopathy on liver post-transplantation outcomes. Int J Hepatol 2013; 2013:952828

8 Wang WL, Yang ZF, Lo CM, Liu CL, Fan ST. Intracerebral hemorrhage after liver transplantation. Liver Transpl 2000; 6:345-348.

9 Murray KF, Carithers RL. AASLD practice guidelines: evaluation of the patient for liver transplantation. Hepatology 2005; 41:1407-1432.

10 Vilstrup H, Amodio P, Bajaj J, Cordoba J, Ferenci P, Mullen KD, et al. Hepatic encephalopathy in chronic liver disease: 2014 practice guideline by AASLD and EASL. Hepatology 2014; 60:715-735.

11 Teasdale G, Jennett B. Assessment of coma and impaired consciousness. A practical scale. Lancet 1974; 2:81-84.

12 Ardizzone G, Arrigo A, Schellino MM, Stratta C, Valzan S, Skurzak S, et al. Neurological complications of liver cirrhosis and orthotopic liver transplant. Transplant Proc 2006; 38:789-792.

13 Amodio P, Biancardi A, Montagnese S, Angeli P, lannizzi P, Cillo U, et al. Neurological complications after orthotopic liver transplantation. Dig Liver Dis 2007; 39:740-747.

14 El-Zanaty F, Way A. Egypt Demographic and Health Survey 2008. Cairo, Egypt: Ministry of Health, El-Zanaty and Associates, and Macro International; 2009.

15 Ministry of Health and Population (Egypt), El-Zanaty and Associates (Egypt) and and ICF International. Egypt Health Issues Survey 2015. Cairo, Egypt and Rockville, Maryland, USA: Ministry of Health and Population and ICF International; 2015.

16 Vizzini G, Asaro M, Miraglia R, Gruttadauria S, Fili D, D’Antoni A, et al. Changing picture of central nervous system complications in liver transplant recipients. Liver Transpl 2011; 17:1279-1285.

17 Kim BS, Lee SG, Hwang S, Park KM, Kim KH, Ahn CS, et al. Neurologic complications in adult living donor liver transplant recipients. Clin Transplant 2007; 21:544-547.

18 Ghaus N, Bohlega S, Rezeig M. Neurological complications in liver transplantation. J Neurol 2001; 248:1042-1048.

19 Amin ME, Atta FE. Neuropsychiatric complications after liver transplantation. Egypt J Psychiatr 2012; 33:57-62.

20 Saner F, Gu Y, Minouchehr S, Ilker K, Fruhauf NR, Paul A, et al. Neurological complications after cadaveric and living donor liver transplantation. J Neurol 2006; 253:612-617.

21 Pujol A, Graus F, Rimola A, Beltrán J, Garcia-Valdecasas JC, Navasa M, et al. Predictive factors of in-hospital CNS complications following liver transplantation. Neurology 1994; 44:1226-1230.

22 Ferreiro JA, Robert MA, Townsend J, Vinters HV. Neuropathologic findings after liver transplantation. Acta Neuropathologica 1992; 84:1-14.

23 Anghel D, Tanasescu R, Campeanu A, Lupescu I, Podda G, Bajenaru O. Neurotoxicity of Immunosuppressive Therapies in Organ Transplantation. Maedica (Buchar) 2013; 8:170-175.

24 Campagna F, Biancardi A, Cillo U, Gatta A, Amodio P. Neurocognitiveneurological complications of liver transplantation: a review. Metab Brain Dis 2010; 25:115-124.

25 Balderramo D, Prieto J, Cárdenas A, Navasa M. Hepatic encephalopathy and post-transplant hyponatremia predict early calcineurin inhibitorinduced neurotoxicity after liver transplantation. Transpl Int 2011; 24:812-819. 